\title{
Survival analysis and competing risk modek of hospital length of stay and discharge destination: the effect of
}

distributional assumptions

Carla Sá - Clara E. Disnuke - Paule Guimaràes

Alstract The hterature on kngth of stay and hospital discharge is often used to inform policy regarding hospital payment and quality. This literature has evolved from the use of ordimary least squares estimation of linear and $\mathrm{kg}$-linear models to the use of survival and conpeting risk model that control for unobserved patient and hospital heterogeneity. However, the authors kend to adopt different distributional assumptions and ofen motivate the choice of specific functional forms for the baseline hamard based on the visual inspection of the hazard rate ploss. We contribuse to this likerature by showing that parameter estimates for patient and hospital characteristics in length of stay modek are particularly sensitive to underlying assumptions regarding the hazard function. Moreover, we demonstrate that the inability to distinguish between competing risks of discharge destination may lead to distortions in the effect of important explanatory variables such as intensive care utilyation.

Keywords Outcomes - Hazard functions - Unobserved heverogeneity

JEL Classification I12

C. Sa

Depotanen of Fonoconis asd NIPE, Universidade do Minha, Braga Portugal

e-mul: angel ia tieeguminbope

C.E. Dianke (ब्खी

Depatment of Hath Adruinistabion asd Policy and CHEPS

Medical Umivesity of South Cardina 151 Rutledge Ave. Bldg B

Charlestoe, SC. 29425, USA

c-muit disarbereresc abu

P. Guimañes

Division of Research, Mooe Sdbool of Business, 1705 College Street.

Colbabia, SC 29208, USA

-mail: guimataesemooresc adv 


\section{Introduction}

Hospital length of stay and discharge policy are key topics of research in both the medical and health economics literature. Length of stay is considered a key indicator of both resource usage and outcomes in the United States (U.S.) and around the world. It is collected for every inpatient stay in countries using some form of the Diagnosis Related Group (DRG) system. DRGs were initially developed in the U.S. but are currently being used in approximately 36 countries for patient information collection, hospital financing or reimbursement for inpatient care (October 2003). This widespread adoption of DRG type systems should facilitate national and international comparisons of inpatient length of stay by DRG. ${ }^{1}$

A survey by Clarke and Rosen (2001) found 548 published articles that analyzed length of stay in some manner between the years 1983 and 1997. Length of stay is the result of a complex process of interaction between patient characteristics, social environment, medical practice, and hospital characteristics but the literature has focused primarily on the effects of patient and hospital characteristics. Moreover, the literature often presents conflicting results regarding the influence of these characteristics on length of stay. ${ }^{2}$

Initial studies of length of stay variation relied on linear and log-linear models estimated by ordinary least squares. However, after Fenn and Davies (1990] showed that variations in hospital discharge policies should be specified as the conditional probability of discharge of the patient using duration models, most subsequent researchers began to focus on the conditional probability of discharge and on the nature of the discharge.

Cutler (1995), Hamilton et al. (1996), and Picone et al. (2003) advanced the methods in length of stay modeling and estimation by emphasizing the need to control for unobserved patient heterogeneity. More importantly, these authors recognized the need to separate out discharge destination and used models of competing risks to account for this. Hamilton and Hamilton (1997) and Ho et al. (2000) suggested that, in addition to unobserved patient heterogeneity, there may also exist important unobserved hospital heterogeneity. They dealt with this potential problem by adding hospital fixed effects controls as a means to control for these unobserved effects. Another relevant contribution to the length of stay literature is due to Clark and Ryan (2002). These authors convincingly argued that, especially when mortality is separate from live discharge, the use of more flexible models (such as the piecewise constant hazard model (PCHM)) for estimation of competing risks, may be a better approach than the parametric methods more commonly used.

The literature on length of stay modeling and estimation has thus evolved from the use of ordinary least squares estimation of linear and log-linear models to the use of survival analysis and competing destination risk models that adjust for unobserved patient heterogeneity and hospital effects. However, in many of these studies, authors often select specific functional forms for the baseline hazard function based on visual inspections of the hazard rate plots, and neglect to model unobserved heterogeneity or to consider the existence of competing risks. We contribute to this literature by showing that estimates of standard survival models and competing risk models for length of stay, with and without controls for

\footnotetext{
${ }^{1}$ While it is the case that reimbursement based on DRGs affects length of stay depending on the mode in which it is used (per patient payment versus hospital budget setting), this issue is beyond the scope of this particular paper. See Dismuke and Guimaraes (2002) for a discussion of the DRG system in Portugal.

${ }^{2}$ For example, Canoodt and Knickman (1984) found that teaching status of hospitals does not significantly influence length of stay, while Burns and Wholey (1991) found that teaching status significantly increases, but Shi (1996) concluded that teaching status significantly reduces length of stay.
} 
unobserved patient heterogeneity and hospital fixed effects can lead to very different conclusions. Moreover, we find consistent with Clark and Ryan (2002), that the PCHM holds great appeal and is a convenient and flexible approach for modeling length of stay.

\section{Econometric models: background}

\subsection{Survival models}

As Fenn and Davies (1990) indicated, inpatient length of stay is most adequately modeled as the probability of discharge given length of stay until that time, so that time until failure (duration or survival) models are most appropriate.

The hazard function, $\lambda$, is a key concept in survival analysis and is defined as the rate of failure at a point in time $t$, given survival until that time:

$$
\lambda(t, \mathbf{x})=\lim _{\mathrm{d} t \rightarrow 0} \frac{\operatorname{Pr}(t \leq T<t+\mathrm{d} t \mid T \geq t, \mathbf{x})}{\mathrm{d} t}
$$

where $T$ denotes the random variable length of stay and $\mathbf{x}$ is a vector of explanatory variables consisting of patient and hospital characteristics. Among the possible alternative interactions between $T$ and $\mathbf{x}$ proposed, the most popular in the length of stay literature is the proportional hazards (PH) specification (Hamilton et al. 1996). In these models, the hazard function is restricted to a multiplicative form so that

$$
\lambda(t, \mathbf{x}, \boldsymbol{\beta}, \boldsymbol{\theta})=\lambda_{0}(t, \boldsymbol{\theta}) \phi(\mathbf{x}, \boldsymbol{\beta})
$$

where $\lambda_{0}$ is the baseline hazard function, $\boldsymbol{\theta}$ is a vector of ancillary parameters characterizing the distribution of $T, \boldsymbol{\beta}$ is a vector of unknown coefficients associated with $\mathbf{x}$ and $\phi(\mathbf{x}, \boldsymbol{\beta})$ is a proportionality factor which does not depend on duration. ${ }^{3}$ With proportional hazards, the effects of the regressors on the conditional probability of failure do not depend on duration. The baseline hazard function summarizes the pattern of duration dependence, and alternative specifications of the baseline function lead to different hazard functions.

\subsubsection{Alternative model formulations}

The impact of the covariates on the hazard function can be estimated using parametric or semi-parametric techniques. The simplest models are parametric models that require restrictive assumptions regarding the functional form of the baseline hazard function such as

$$
\begin{gathered}
\text { Exponential: } \lambda(t, \mathbf{x}, \boldsymbol{\beta})=\exp \left(\mathbf{x}^{\prime} \boldsymbol{\beta}\right), \\
\text { Weibull: } \lambda(t, \mathbf{x}, \boldsymbol{\beta}, \alpha)=\alpha t^{\alpha-1} \exp \left(\mathbf{x}^{\prime} \boldsymbol{\beta}\right) .
\end{gathered}
$$

The exponential model assumes a constant baseline hazard for each patient while the baseline hazard for the Weibull model is strictly increasing or decreasing depending on the value of $\alpha .{ }^{4}$ Other baseline hazard specifications are possible but proportional hazards

\footnotetext{
${ }^{3} \phi(\mathbf{x}, \boldsymbol{\beta})$ is a non-negative function of the covariates. As typically done in the literature we let this function be exponential and will henceforth replace it by $\exp \left(\mathbf{x}^{\prime} \boldsymbol{\beta}\right)$.

4 The parameter $\alpha$ assumes only positive values. If $\alpha>1$ then the hazard function increases monotonically, if $\alpha<1$ then it decreases monotonically and if $\alpha=1$ the model collapses to the exponential case.
} 
models can be estimated with greater flexibility by assuming a less rigid functional form for the hazard function such as the piecewise constant hazard specification. Under the piecewise constant hazard assumption, the time period of interest is divided into $M$ intervals, such that $\left(c_{m-1}, c_{m}\right]$, where $c_{m}$ are known constants, and $m=0,1, \ldots, M-1, c_{0}=0$ and $c_{M}=\infty$. The choice of the intervals should be made in such a manner that they become closer when the hazard function varies more rapidly and become further apart when variations in the hazard function are slower (Rodriguez 2000). There then exist $M$ unknown constants, $\phi_{m}$, which represent different values of the baseline hazard function for each of the pre-specified intervals:

$$
\text { Piecewise-constant: } \lambda\left(t, \mathbf{x}, \boldsymbol{\beta}, \varphi_{m}\right)=\exp \left(\varphi_{m}\right) \exp \left(\mathbf{x}^{\prime} \boldsymbol{\beta}\right), \quad \varphi_{m}=1, \ldots, M .
$$

The above duration models are easily estimated by maximum likelihood. The contribution of complete observations to the likelihood function is given by the density function for the length of stay, $f$, while the contribution of incomplete (right censored) durations ${ }^{5}$ is given by the survival function, $S$. Regardless of the functional form chosen for the hazard function the density and survival functions can be expressed in terms of the hazard function: ${ }^{6}$

$$
\begin{gathered}
S\left(t_{i}, \mathbf{x}_{i}, \boldsymbol{\beta}, \boldsymbol{\theta}\right)=\exp \left(-\int_{0}^{t} \lambda\left(t_{i}, \mathbf{x}_{i}, \boldsymbol{\beta}, \boldsymbol{\theta}\right) \mathrm{d} t\right), \\
f\left(t_{i}, \mathbf{x}_{i}, \boldsymbol{\beta}, \boldsymbol{\theta}\right)=\lambda\left(t_{i}, \mathbf{x}_{i}, \boldsymbol{\beta}, \boldsymbol{\theta}\right) S\left(t_{i}, \mathbf{x}_{i}, \boldsymbol{\beta}, \boldsymbol{\theta}\right) .
\end{gathered}
$$

The log-likelihood function is then given by:

$$
\ln L=\sum_{i=1}^{n} d_{i} \ln f\left(t_{i}, \mathbf{x}_{i}, \boldsymbol{\beta}, \boldsymbol{\theta}\right)+\sum_{i=1}^{n}\left(1-d_{i}\right) \ln S\left(t_{i}, \mathbf{x}_{i}, \boldsymbol{\beta}, \boldsymbol{\theta}\right)
$$

where $n$ is the total number of observations and $d_{i}$ is 1 if the duration for the $i$ th observation is complete, and 0 if it is censored.

The Cox PH model is the most commonly used semi-parametric duration model (Jones 2000). Cox suggested a likelihood procedure (partial likelihood) to estimate the relationship between the hazard rate and explanatory variables in the following general proportional hazards model:

$$
\operatorname{Cox} \mathrm{PH}: \lambda(t, \mathbf{x}, \boldsymbol{\beta}, \boldsymbol{\theta})=\lambda_{0}(t, \boldsymbol{\theta}) \exp \left(\mathbf{x}^{\prime} \boldsymbol{\beta}\right) .
$$

In Cox's model we do not need to make any assumptions about the functional form of the baseline hazard function, $\lambda_{0}$. However, since the partial likelihood approach discards information regarding actual failure times and uses only their rank order, the efficiency of the estimates obtained by this approach is reduced.

\footnotetext{
5 A duration is right censored if all that is known about that duration is that it lasted longer than a certain time.

${ }^{6}$ So far, we avoided using the subscript $i$ for individual observations in order to facilitate the exposition. We only use it in presenting the likelihood function.
} 


\subsubsection{Unobserved heterogeneity}

It is difficult for the researcher to control for all relevant patient and hospital characteristics. However, unlike the linear regression case, duration models that do not account for unobservable heterogeneity will produce biased estimates of duration dependence (Jones 2000; Elbers and Ridder 1982). A common way of approaching this problem is by letting the hazard function of a patient be modified by a proportionality factor (frailty). If we let $v>0$ represent non-measured heterogeneity then the hazard function becomes:

$$
\lambda(t, \mathbf{x}, \boldsymbol{\beta}, \boldsymbol{\theta})=\lambda_{0}(t, \boldsymbol{\theta}) \exp \left(\mathbf{x}^{\prime} \boldsymbol{\beta}\right) v .
$$

Thus, for $v$ larger than 1 , patients will have a higher risk of failure while the opposite is true for values of $v$ below 1 . The proportionality factor $v$ may be patient specific (unshared frailty) or hospital specific (shared frailty). Commonly, $v$ is assumed to be a realization of a random variable $V$, which has known distribution and is uncorrelated with the other covariates. When $v$ is a shared frailty it may be possible to model it as a fixed effect.

An alternative approach to deal with unobservable heterogeneity is to use robust variance estimates. This approach is less common in the literature, most likely because its validity is conditional on the assumption that the hazard function is correctly specified (Cameron and Trivedi 2005). A related result is that of Clegg et al. (2000). These authors studied the application of "population-averaged" marginal regression models to correlated censored duration data. Their results indicated that misspecification of the marginal hazards model in a marginal regression framework will lead to inconsistent estimators in general.

\subsubsection{Patient unobserved effects Hamilton et al. (1996) emphasized the potential} problem of patient unobserved heterogeneity in models that have patient characteristics as regressors. Lancaster (1990) presented some examples of possible distributions for $V$, including the Gamma distribution. The choice of the Gamma distribution for $V$ is motivated by computational convenience because, for particular baseline distributions, it is possible to obtain a closed form solution when $v$ is integrated out of the expression (10). If we assume that the baseline function $\lambda_{0}$ follows a Weibull distribution, and that $V$ follows a Gamma distribution with an expected value of 1 and variance of $\sigma^{2}$, a Gamma mixing of the Weibull function, commonly known as the Burr model, is obtained. The resulting (mixed) hazard function is given by:

$$
\lambda(t, \mathbf{x}, \boldsymbol{\beta}, \sigma, \alpha)=\frac{\exp \left(\mathbf{x}^{\prime} \boldsymbol{\beta}\right) \alpha t^{\alpha-1}}{1+\sigma^{2} \exp \left(\mathbf{x}^{\prime} \boldsymbol{\beta}\right) t^{\alpha}} .
$$

Cutler (1995) and Hamilton et al. (1996) used an alternative method developed by Heckman and Singer (1984) to deal with patient unobserved heterogeneity. Here $V$ is assumed to have a discrete distribution so that $g\left(v_{k}\right)=\operatorname{Pr}\left(V=v_{k}\right)=p_{k}, k=1,2, \ldots, K$ where $K$ is the number of support points of the distribution. For ease of exposition, let us consider $K=2$, that is, assume that $V$ takes two different values, $v_{1}$ and $v_{2}$, with probabilities $p$ and $1-p$, respectively. If we consider that the no-frailty hazard component has a proportional hazards form with a Weibull baseline hazard, then the mixed hazard function is given by

$$
\begin{aligned}
\lambda\left(t, \mathbf{x}, \boldsymbol{\beta}, v_{1}, v_{2}, p\right) & =p \lambda\left(t, \mathbf{x}, \boldsymbol{\beta}, v_{1}\right)+(1-p) \lambda\left(t, \mathbf{x}, \boldsymbol{\beta}, v_{2}\right) \\
& =p \alpha t^{\alpha-1} \exp \left(\mathbf{x}^{\prime} \boldsymbol{\beta}+v_{1}\right)+(1-p) \alpha t^{\alpha-1} \exp \left(\mathbf{x}^{\prime} \boldsymbol{\beta}+v_{2}\right) .
\end{aligned}
$$


With the Heckman and Singer approach to patient unobserved heterogeneity it is relatively simple to employ different formulations for the baseline hazard. For example, if the PCHM is assumed for the baseline hazard then the mixed hazard function becomes:

$$
\begin{aligned}
\lambda\left(t, \mathbf{x}, \boldsymbol{\beta}, \varphi_{m}, v_{1}, v_{2}, p\right) & =p \lambda\left(t, \mathbf{x}, \boldsymbol{\beta}, \varphi_{m}, v_{1}\right)+(1-p) \lambda\left(t, \mathbf{x}, \boldsymbol{\beta}, \varphi_{m}, v_{2}\right) \\
& =p \exp \left(\varphi_{m}\right) \exp \left(\mathbf{x}^{\prime} \boldsymbol{\beta}+v_{1}\right)+(1-p) \exp \left(\varphi_{m}\right) \exp \left(\mathbf{x}^{\prime} \boldsymbol{\beta}+v_{2}\right) .
\end{aligned}
$$

All of the above models are easily estimated by maximum likelihood using the general expression for the log-likelihood shown in (8).

2.1.2.2 Hospital unobserved effects An important issue that has to be addressed when analyzing hospital length-of-stay, is the issue of possible clustering of length-of-stay within hospitals. There may be unobservable hospital effects that are likely to affect discharge probabilities in a systematic manner. From a technical perspective this situation is similar to patient heterogeneity with the caveat that patients from the same hospital have identical unobserved hospital effects (shared frailty) and so the hazard function becomes:

$$
\lambda(t, \mathbf{x}, \boldsymbol{\beta}, \boldsymbol{\theta})=\lambda_{0}(t, \boldsymbol{\theta}) \exp \left(\mathbf{x}^{\prime} \boldsymbol{\beta}\right) v_{\mathrm{h}},
$$

where the index $\mathrm{h}$ (for hospital) is added to emphasize that this value is common to all patients from the same hospital. That is, that $v_{h}$ is a "hospital shared frailty". In this latter situation it becomes possible to avoid the specification of a distribution for the frailty and to simply add a set of hospital dummy variables to the explanatory variables included in the model (hospital fixed effects). This approach has the advantage of fully accounting for all the variation in length of stay that can be attributed exclusively to differences across hospitals. However, this approach is unsuitable for a researcher interested in the impact of specific hospital characteristics on length of stay because all variables that account for inter-hospital variability are absorbed by the hospital dummy variables. Alternatively, hospital effects may be modeled as random, in which case $V_{\mathrm{h}}$ is assumed to follow a known random distribution. Now, it becomes possible to obtain estimates for the impact of specific hospital characteristics in the hazard function. Again, the most common approach is to assume that $V_{\mathrm{h}}$ follows a Gamma distribution. Just as with patient unobserved heterogeneity, if we assume that the baseline hazard is Weibull, we obtain a closed-form solution for the likelihood function (Hougaard 1984). Interestingly enough, as shown in Therneau and Grambsch (2000), it is possible to estimate the parameters of a Cox PH regression model, under the assumption of a shared Gamma frailty.

\subsection{Competing risk models}

The necessity to distinguish between different types of duration failure resulted in extensions of the models presented above. As Gooley et al. (1999) explain, when several risks are present, the number of failures from any competing risk will influence the number of failures from the cause being analyzed and thus the estimate of the probability of failure of that cause. This is so because failures from any competing risk reduce the number of patients at risk of failure from the cause under analysis. In length of stay models, the 
implication is that if multiple destinations are possible upon discharge from the hospital, then the number of discharges to a particular destination will influence the estimate of the probability of leaving the hospital. Consider, for example, three possible discharge destinations: died in the hospital, home, and transferred to another hospital. In this case, as more individuals die in the hospital, there are fewer individuals at risk to be transferred to another hospital.

For each individual in a competing risk model, a variable indicating the type of failure is specified, $J$, with the values $1, \ldots, k$ in addition to the random duration variable, $T$. The simplest form of this model considers only one period of duration that terminates when an individual leaves for one of $k$ possible states. These states, mutually exclusive and exhaustive, are designated by the index $j$, so that $j=1, \ldots, k$. Consider the existence of $k$ random variables, $T^{(1)}, T^{(2)}, \ldots, T^{(k)}$, one for each destination, interpreted as latent duration. These are imaginary time periods used in the construction of the models whereby $T^{(j)}$ is the time to failure to state $j$, eliminating all other possible states. At each moment, entry into a certain state is dictated by the smallest latent time period, i.e. by the smallest $T^{(j)}$. Mathematically, this can be specified in the following manner: $T=\min \left[T^{(1)}, \ldots, T^{(k)}\right]$ and $J=j$, if $T=T^{(j)}$. For each individual, only one $T^{(j)}$ is observed and others are considered censored. Under the assumption that the random variables $T^{(1)}, \ldots, T^{(k)}$ are independent, the model is effectively that of independent competing risks.

It is possible to estimate conditional and unconditional probability functions that characterize the variables $T$ and $J$. The expression

$$
\lambda_{j}(t, \mathbf{x})=\lim _{\mathrm{d} t \rightarrow 0} \frac{\operatorname{Pr}(t \leq T<t+\mathrm{d} t, J=j \mid T \geq t, \mathbf{x})}{\mathrm{d} t}
$$

is the transition intensity into state $j$. These functions, often designated as cause-specific hazard functions, can be empirically interpreted as the fraction of survivors at time $t$ that subsequently leave for state $j$. Assuming a proportional hazards specification, the causespecific hazard functions can be defined as

$$
\lambda_{j}\left(t, \mathbf{x}, \boldsymbol{\beta}_{j}, \alpha_{j}\right)=\lambda_{0 j}\left(t, \alpha_{j}\right) \exp \left(\mathbf{x}^{\prime} \boldsymbol{\beta}_{j}\right), j=1, \ldots, k,
$$

where the risk-specific baseline hazard function is $\lambda_{0 j}\left(t, \alpha_{j}\right)$ and, $\boldsymbol{\beta}_{j}$ and $\alpha_{j}$ are allowed to freely vary for the $k$ types of failure. As before, alternative distributions for the causespecific baseline hazard lead to different cause-specific hazard functions. For example, if a Weibull baseline hazard is assumed, then the hazard function is

$$
\lambda_{j}\left(t, \mathbf{x}, \alpha_{j}, \boldsymbol{\beta}_{j}\right)=\alpha_{j} t^{\alpha_{j}-1} \exp \left(\mathbf{x}^{\prime} \boldsymbol{\beta}_{j}\right),
$$

an expression identical to (4). Note, however, that we can now estimate a set of coefficients for each one of the competing risks. Similarly, we can recast all of the other models presented earlier, including those that account for unobserved heterogeneity, as independent competing risks models.

The log-likelihood function is now expressed as, ${ }^{7}$

$$
\ln L=\sum_{j=1}^{k}\left[\sum_{i=1}^{n} d_{i} \ln f\left(t_{i}, \boldsymbol{\beta}_{j}, \mathbf{x}_{i}, \boldsymbol{\theta}_{j}\right)+\sum_{i=1}^{n}\left(1-d_{i}\right) \ln S\left(t_{i}, \boldsymbol{\beta}_{j}, \mathbf{x}_{i}, \boldsymbol{\theta}_{j}\right)\right] .
$$

\footnotetext{
7 Again, we only use the individual subscript in presenting the likelihood function, but leave it out for the remainder of the text.
} 
Furthermore, inspection of the log-likelihood expression makes it obvious that independent competing risk models may be estimated as separate models, one for each risk, with observations for other risks treated as censored. This logic applies also to the Cox PH model which can be estimated for competing risks, with or without (shared) frailty. ${ }^{8}$

\subsubsection{Identification issues}

The introduction of unobserved heterogeneity into survival models raises a natural question of identification. The problem may be expressed as follows: are the baseline hazard and heterogeneity distributions unique, given the data? That is not necessarily the case. Cameron and Trivedi (2005) summarize the two main positions regarding this issue: at one end there are those who argue that one should favor nonparametric specifications of heterogeneity such as the Heckman and Singer approach while, at the other end there are those who defend that parametric specifications of unobserved heterogeneity are innocuous provided the baseline is correctly specified. Identifiability of the frailty distribution parameters requires that the frailty variable, $V$, be bounded, with expected mean of one and finite variance.

Identification of the joint distribution of all latent durations, $T^{(k)}$, is also a major issue in competing risks models (see, for instance, Bedford and Lindqvist (2004)). However, Heckman and Honoré (1989) have shown that under non-restrictive assumptions, independent competing risks models with proportional hazards specification are identified, provided they have covariates.

\section{Model specification and empirical strategy}

\subsection{Data and variables}

We test the models described in Sect. 2 for length of stay outcomes using inpatient claims data for patients with cerebrovascular disorders from Portugal that were hospitalized at any time during the years of 1992 and 1993 . The final sample consists of 34,250 observations. ${ }^{9}$

Length of stay is the (continuous) duration variable, $T .{ }^{10}$ For patients who are admitted and discharged on different days, duration is calculated as the difference between the discharge and admissions dates. Patients are required to have a stay of at least $24 \mathrm{~h}$ in order to be classified as inpatient and attributed a DRG. Discharge destination, $J$, is equal to zero

\footnotetext{
${ }^{8}$ For a more detailed discussion on competing risk models, see, for instance, Cox (1959), David and Moeschberger (1978), and Prentice et al. (1978).

9 The Instituto de Gestão Informatica e Financeira, the entity responsible for the management of the information technology and financial resources of the Portuguese Ministry of Health, provided all public hospital discharges classified into DRG 14, Cerebrovascular Disorders Except Transient Ischemic Attack, for the January 1992-December 1994 time period. DRG 14-Cerebrovascular Disorders includes the following ICD-9-CM diagnosis codes: 430 Hemorrhage, subarachnoid; 431 Hemorrhage, intercerebral; 432 Hemorrhage, intracranial, other and unspecified; 433.01 Occlusion and stenosis, basilar artery with cerebral infarction; 433.11 Occlusion and stenosis, cartoid artery with cerebral infarction; 433.21 Occlusion and stenosis, vertebral artery, with cerebral infarction; 433.31 Occlusion and stenosis, multiple and bilateral arteries, with cerebral infarction; 433.81 Occlusion and stenosis, other specified precerebral artery, with cerebral infarction; 433.91 Occlusion and stenosis, unspecified precerebral artery, with cerebral infarction; 434.01 Thrombosis, cerebral, with cerebral infarction.

${ }^{10}$ Although in practice, time is always measured in discrete units, "when these units are very small, it is usually acceptable to treat time as if it were measured on a continuous scale" Allison (1984), p. 14.
} 
Table 1 Distribution of length of stay (LOS) and discharge destination

\begin{tabular}{lllllll}
\hline LOS $^{\mathrm{a}}$ & $N$ & $\%$ & Censored & Home & Transferred & Died \\
\hline 1 & 4,724 & 13.79 & 131 & 1,291 & 1,799 & 1,503 \\
2 & 2,213 & 6.46 & 56 & 746 & 483 & 928 \\
$3-6$ & 6,321 & 18.46 & 134 & 3,528 & 500 & 2,159 \\
$7-12$ & 8,477 & 24.75 & 68 & 6,642 & 332 & 1,435 \\
$13-20$ & 6,396 & 18.68 & 33 & 5,344 & 246 & 773 \\
$21-30$ & 3,471 & 10.13 & 23 & 2,856 & 149 & 443 \\
31 or + & 2,648 & 7.73 & 36 & 2,040 & 92 & 480 \\
Total & 34,250 & 100 & 481 & 22,447 & 3,601 & 7,721 \\
\hline
\end{tabular}

a The intervals in the first column correspond to the steps in the PCHM

if the observation is censored, 1 if the individual is discharged home, 2 if the individual is transferred to another hospital and 3 if the individual dies in the hospital. Furthermore, for each individual, a binary variable, $d$, was created which is equal to 1 if the observation is complete and 0 in the case where the duration is censored. Patients discharged against medical advice are classified as censored since the outcome of these patients is considered to be beyond the control of the hospital. For more detailed information about the duration and discharge destination, see Table 1.

The explanatory variables are thus typically divided into patient and hospital characteristics (see Table 2 for a more detailed description of the variables). Patient characteristics used as regressors include a binary variable for gender (Female), age (Age), whether or not the patient received intensive care $(I C U)$, whether or not the patient received treatment in more than one service (e.g. internal medicine, neurology) (Service), and a measure of patient severity, the Charlson Index Score ${ }^{11}$ (Charlson). We also include whether or not the patient received a Computerized Tomography Scan ${ }^{12}$ (CTScan). Hospital characteristics include variables that provide an administrative classification of hospitals ${ }^{13}$; level (Level1) and district (District), whether the hospital is a central nonteaching hospital (NonTeach), the logarithm of number of beds (LnBeds), and the hospital case-mix (CaseMix) for the year in which the discharge took place. ${ }^{14}$

11 The Charlson Index contains 19 categories of comorbidity, which are primarily defined using ICD-9-CM diagnoses codes (a few procedure codes are also employed). Each category has an associated weight, taken from the original Charlson paper (1987), which is based on the adjusted risk of 1-year mortality. The overall comorbidity score reflects the cumulative increased likelihood of 1-year mortality; the higher the score, the more severe the burden of comorbidity.

12 According to practice guidelines, Computerized Tomography of the head is critical for the emergent evaluation of patients with acute stroke. It is important for excluding or documenting inter-cranial hemorrhaging as the stroke mechanism and to identify other features that directly or indirectly impact the diagnostic evaluation and management of stroke (Culebras et al. 1997).

13 Portuguese hospitals are classified as either central, district or level one depending on the availability of specialties. Central hospitals generally provide all specialties, district hospitals a moderate number and level one, a basic level of specialties. The level of technological capacity also varies from greatest in central to least in the level one hospitals.

14 The Casemix variable is a measure of the severity of all patients treated in a given hospital. To compute this variable, each discharge in a hospital is weighted with the relative weight of its DRG, and the weighted sum of all discharges is divided by the number of discharges in that hospital. 
Table 2 Definition of variables and descriptive statistics

\begin{tabular}{llll}
\hline Variable & Definition & Mean & Standard deviation \\
\hline$T$ & LOS (days) & 12.843 & 16.912 \\
$D$ & 1 if the observation is complete & 0.986 & \\
$J$ & 0 if the observation is censored & 0.014 & \\
& 1 if patient discharged home & 0.655 & \\
& 2 if patient is transferred to another hospital & 0.105 & \\
Patient characteristics & 0.225 & \\
Female & 1 if female & 0.490 & \\
Age & Age in years & 70.002 & \\
ICU & 1 if patient received intensive care & 0.012 & \\
Service & 1 if treated in more than 1 service & 0.033 & \\
Charlson & Charlson index of severity & 1.466 & \\
CTScan & 1 if patient received a CAT scan & 0.352 & \\
Hospital characteristics & & \\
Level1 & 1 if level one hospital & 0.137 & \\
District & 1 if district hospital & 0.489 & \\
NonTeach & 1 if central non-teaching & 0.137 & \\
LnBeds & Log number of beds & 5.784 & 0.930 \\
CaseMix & Case-mix in the year of admission & 1.014 & 0.243 \\
\hline
\end{tabular}

The regressors used are the same in all specifications. Thus,

$$
\begin{array}{r}
x^{\prime} \beta=\beta_{1} \text { Female }+\beta_{2} \text { Age }+\beta_{3} \text { ICU }+\beta_{4} \text { Service }+\beta_{5} \text { Charlson }+\beta_{6} \text { CTScan } \\
+\beta_{7} \text { Level } 1+\beta_{8} \text { District }+\beta_{9} \text { NonTeach }+\beta_{10} \text { LnBeds }+\beta_{11} \text { CaseMix }
\end{array}
$$

where, depending on the specification, the constant may be explicitly added to the model or defined implicitly by a set of dummy variables. Coefficient estimates are then interpreted as the impact of each variable on the (conditional) probability of discharge and consequently on the length of stay. For example, a positive estimate for $\beta_{2}$ indicates that, everything else constant, older individuals show higher discharge probabilities and hence are less likely to stay longer in the hospital.

\section{Results}

All models were estimated using the statistical software STATA version $8.0{ }^{15}$ A comparison of the estimates from the basic survival models, is shown in Table 3 .

Results from all specifications in Table 3 are similar both in terms of the coefficients' signs and significance. The estimates from the Exponential and Weibull model are practically identical. This is not surprising given that the values for the log-likelihoods of

15 Most models were estimated using the STREG command in Stata. The exception were the Cox models that were estimated with the STCOX procedure and models with Heckman and Singer heterogeneity that required programming the likelihood function using ML commands. A list with the Stata code used in this paper is available at http:// www2.eeg.uminho.pt/economia/cangelica/downloads/stata_commands.pdf. 
Table 3 Basic survival models ${ }^{\mathrm{a}}$

\begin{tabular}{|c|c|c|c|c|}
\hline Variables & Exponential & Weibull & $\mathrm{PCHM}^{\mathrm{b}}$ & Cox \\
\hline Female & $-0.054 *(0.011)$ & $-0.055 *(0.011)$ & $-0.051 *(0.011)$ & $-0.049 *(0.011)$ \\
\hline Age & $0.002 *(0.000)$ & $0.002 *(0.000)$ & $0.002 *(0.000)$ & $0.001 *(0.000)$ \\
\hline $\mathrm{ICU}$ & $-0.224 *(0.051)$ & $-0.224 *(0.051)$ & $-0.217 *(0.051)$ & $-0.230 *(0.051)$ \\
\hline Service & $-0.521 *(0.031)$ & $-0.523 *(0.031)$ & $-0.466 *(0.031)$ & $-0.459 *(0.031)$ \\
\hline Charlson & $-0.032 *(0.002)$ & $-0.032 *(0.002)$ & $-0.028 *(0.002)$ & $-0.026^{*}(0.002)$ \\
\hline CTScan & $-0.104 *(0.012)$ & $-0.104 *(0.012)$ & $-0.121 *(0.012)$ & $-0.134 *(0.012)$ \\
\hline level1 & $-1.411 *(0.042)$ & $-1.415 *(0.042)$ & $-1.266^{*}(0.041)$ & $-1.260 *(0.041)$ \\
\hline District & $-0.966^{*}(0.029)$ & $-0.970 *(0.029)$ & $-0.861 *(0.028)$ & $-0.854 *(0.028)$ \\
\hline NonTeach & $-0.545^{*}(0.021)$ & $-0.548 *(0.021)$ & $-0.481 *(0.021)$ & $-0.477 *(0.021)$ \\
\hline LnBeds & $-0.283 *(0.013)$ & $-0.284 *(0.013)$ & $-0.254 *(0.013)$ & $-0.253 *(0.013)$ \\
\hline CaseMix & $-0.882 *(0.038)$ & $-0.886 *(0.038)$ & $-0.772 *(0.037)$ & $-0.756 *(0.037)$ \\
\hline Constant & $0.736^{*}(0.105)$ & $0.732 *(0.105)$ & & \\
\hline$\alpha$ & & $1.005(0.004)$ & & \\
\hline $\log L$ & -51695.3 & -51695 & -50689 & -319341.4 \\
\hline
\end{tabular}

a Significance at the 1,5 and $10 \%$ level is indicated with *,** and $* * *$, respectively. Standard errors are given in parentheses

b For the PCHM seven parameters (not reported) were estimated along with all the other coefficients

the two models are very similar. This means that we cannot reject the hypothesis that $\alpha=1$ for the Weibull model, and thus that the baseline hazard is constant. ${ }^{16}$ On the other hand, the results of the PCHM are quite similar to those of the Cox PH model, suggesting that the step function used for the baseline hazard approximates well the unknown baseline hazard. However, the hypothesis of a constant baseline hazard function is rejected for the PCHM. ${ }^{17}$ This inconsistency may suggest that other problems, such as the presence of some form of unobserved heterogeneity, may be affecting the results.

Hence, we next estimate the models that take into account unobserved patient heterogeneity. These are shown in Table 4. In general the signs and magnitude of the coefficients are similar to those presented in Table 3. However, the volatility of the estimates is higher, especially with respect to the coefficients on hospital characteristics. One of the known consequences of neglected heterogeneity in proportional hazards models is that the hazard rate is underestimated (Cameron et al. 2005). Note that with the introduction of Gamma heterogeneity, the parameter of the Weibull distribution is now clearly above 1, indicating the existence of positive duration dependence. On the other hand, the estimate for the variance of the Gamma distribution is much smaller in the piecewise constant hazard specification, suggesting that misspecification of the baseline hazard may be an issue with the Weibull model.

Up to now, we have not yet accounted for hospital heterogeneity. To do this we introduce hospital fixed effects ${ }^{18}$ to the models presented in Table 4 . This approach leads to

16 The parameter on duration dependence is not significantly different from 1 at the $1 \%$ level.

17 A likelihood ratio test of the hypothesis of equality for the seven ancillary parameters of the piecewise constant model, with a chi-squared test statistic of 2,013.4, is rejected at the $1 \%$ significance level.

18 There are 78 hospitals in our data, so that models with hospital fixed effects include 77 additional dummy variables. 
Table 4 Basic survival models with unobserved patient heterogeneity ${ }^{a}$

\begin{tabular}{|c|c|c|c|c|}
\hline Variables & $\begin{array}{l}\text { Weibull with Gamma } \\
\text { het. }\end{array}$ & $\begin{array}{l}\text { Weibull with H.S. } \\
\text { het. }\end{array}$ & $\begin{array}{l}\text { PCHM with Gamma } \\
\text { het. }^{\text {b }}\end{array}$ & $\begin{array}{l}\text { PCHM with H.S. } \\
\text { het. }^{\text {b }}\end{array}$ \\
\hline Female & $-0.072 *(0.014)$ & $-0.060 *(0.012)$ & $-0.059^{*}(0.012)$ & $-0.052 *(0.012)$ \\
\hline Age & $0.001 * *(0.001)$ & $0.002 *(0.001)$ & $0.000 *(0.001)$ & $0.000 *(0.001)$ \\
\hline ICU & $-0.369^{*}(0.066)$ & $-0.287 *(0.056)$ & $-0.269^{*}(0.057)$ & $-0.250 *(0.054)$ \\
\hline Service & $-0.653^{*}(0.041)$ & $-0.557 *(0.035)$ & $-0.544 *(0.036)$ & $-0.517 *(0.034)$ \\
\hline Charlson & $-0.041 *(0.003)$ & $-0.033^{*}(0.003)$ & $-0.031 *(0.003)$ & $-0.027 *(0.002)$ \\
\hline CTScan & $-0.230 *(0.016)$ & $-0.186 *(0.014)$ & $-0.166^{*}(0.014)$ & $-0.165^{*}(0.013)$ \\
\hline Level1 & $-2.146 *(0.055)$ & $-1.795 *(0.048)$ & $-1.442 *(0.047)$ & $-1.391 *(0.044)$ \\
\hline District & $-1.455^{*}(0.036)$ & $-1.227 *(0.033)$ & $-0.971 *(0.032)$ & $-0.933 *(0.030)$ \\
\hline NonTeach & $-0.700 *(0.027)$ & $-0.624 *(0.024)$ & $-0.528^{*}(0.023)$ & $-0.515^{*}(0.022)$ \\
\hline LnBeds & $-0.480 *(0.017)$ & $-0.392 *(0.015)$ & $-0.297 *(0.015)$ & $-0.288 *(0.014)$ \\
\hline CaseMix & $-1.161 *(0.049)$ & $-1.004 *(0.043)$ & $-0.839 *(0.042)$ & $-0.789 *(0.040)$ \\
\hline Constant & $2.194 *(0.134)$ & & & \\
\hline$\alpha$ & $1.285(0.009)$ & $1.137(0.006)$ & & \\
\hline$\sigma^{2}$ & $0.362(0.012)$ & & $0.124(0.011)$ & \\
\hline$v_{1}$ & & $-0.287(0.131)$ & & $-0.563(0.135)$ \\
\hline$v_{2}$ & & $1.486(0.119)$ & & $1.201(0.111)$ \\
\hline$P$ & & $0.040(0.004)$ & & $0.018(0.003)$ \\
\hline $\log L$ & -50684.9 & -50761.6 & -50595.4 & -50398.4 \\
\hline
\end{tabular}

a Significance at the 1, 5 and $10 \%$ level is indicated with $* * *$ and $* * *$, respectively. Standard errors are given in parentheses

b For the PCHM seven parameters (not reported) were estimated along with all the other coefficients

a marked improvement on the log-likelihoods even after adjusting for degrees of freedom. ${ }^{19}$

Nevertheless, even though we are now accounting for hospital unobserved heterogeneity we are still observing similar results as reported for the model that accounted exclusively for patient unobserved heterogeneity: the Weibull model is indicating positive duration dependence, the variance of the Gamma distribution drops significantly when we allow for a flexible baseline hazard. The main difference is that the ICU variable changes signs becoming positive and is significant in the PCHM with Heckman-Singer heterogeneity. Normally, one would expect ICU usage to increase length of stay so this result appears counterintuitive. ${ }^{20}$

We then estimate independent competing risks models for the probability of discharge, conditional on the manner in which the patient left the hospital. Three possible destinations of the patient were considered: discharged home alive, transferred to another hospital, and in-hospital death. Although we estimated several variants of the competing risks models

\footnotetext{
19 The Akaike information criterion (AIC) for the models in Table 4 is 101,397.8, 101,553.2, 101,228.8 and $100,838.8$ versus $97,119.4,97,113.4,98,176.8$ and 97,554.2 for Table 5. Similarly, the Bayesian information criterion (BIC) is 101,516, 101,679.8, 101,389.2 and 101,016 versus 97,837, 97,847.8, 98,945 and 98,339.3. ${ }^{20}$ We also estimated models that treated the hospital effects as random, namely the Weibull, piecewiseconstant hazard and Cox model all with Gamma shared frailty. All models pointed to the existence of unobserved hospital heterogeneity but provided estimates that were similar in sign and significance to models that accounted exclusively for unobserved patient heterogeneity.
} 
Table 5 Survival models with unobserved patient heterogeneity and hospital fixed effects ${ }^{\mathrm{a}}$

\begin{tabular}{lllll}
\hline Variables & $\begin{array}{l}\text { Weibull with Gamma } \\
\text { het. }\end{array}$ & $\begin{array}{l}\text { Weibull with H.S. } \\
\text { het. }\end{array}$ & $\begin{array}{l}\text { PCHM with Gamma } \\
\text { het. }^{\text {b }}\end{array}$ & $\begin{array}{l}\text { PCHM with H.S. } \\
\text { het. }^{\text {b }}\end{array}$ \\
\hline Female & $-0.072 *(0.015)$ & $-0.062 *(0.012)$ & $-0.061 *(0.013)$ & $-0.052 *(0.012)$ \\
Age & $0.000(0.001)$ & $0.000(0.001)$ & $0.001(0.001)$ & $0.001(0.000)$ \\
ICU & $0.100(0.080)$ & $0.042(0.066)$ & $0.104(0.069)$ & $0.072 *(0.064)$ \\
Service & $-0.932 *(0.045)$ & $-0.776 *(0.039)$ & $-0.753 *(0.039)$ & $-0.704 *(0.037)$ \\
Charlson & $-0.031^{*}(0.003)$ & $-0.024 *(0.003)$ & $-0.023 *(0.003)$ & $-0.020 *(0.003)$ \\
CTScan & $-0.444 *(0.020)$ & $-0.332 *(0.016)$ & $-0.304 *(0.018)$ & $-0.289 *(0.015)$ \\
$\alpha$ & $1.397(0.010)$ & $1.218(0.006)$ & & \\
$\sigma^{2}$ & $0.392(0.013)$ & & $0.136(0.012)$ & \\
$v_{1}$ & & $-4.946(0.069)$ & & $-4.038(0.083)$ \\
$v_{2}$ & & $-2.789(0.043)$ & & $-2.002(0.041)$ \\
$P$ & & $0.027(0.002)$ & & $0.015(0.002)$ \\
LogL & -48474.7 & -48469.7 & -48997.4 & -48684.1 \\
\hline
\end{tabular}

a Significance at the 1,5 and 10\% level is indicated with *,** and ***, respectively. Standard errors are given in parentheses

b For the PCHM seven parameters (not reported) were estimated along with all the other coefficients

we only report results for two of these models: the Weibull and PCHM with Gamma patient heterogeneity and hospital fixed effects.

The estimates in Tables 6-8 show the relevance of distinguishing between different types of discharge in competing risk models since they clarify some of the estimates obtained for the previous models. For example, in the survival models estimated earlier age appeared to have a positive impact in the probability of discharge but the competing risks version of this model shows age reducing the conditional probability of discharge home and transfer to another acute care facility.

The piecewise constant hazard competing risks model is our preferred one. Most notably, the variance for the patient unobserved random effects is much smaller than that

Table 6 Competing risks models: discharged home ${ }^{\mathrm{a}}$

\begin{tabular}{lll}
\hline Variables & Weibull with Gamma het. and hospital FE & PCHM with Gamma het. and hospital FE \\
\hline Female & $-0.136^{*}(0.021)$ & $-0.072^{*}(0.018)$ \\
Age & $-0.044^{*}(0.001)$ & $-0.004^{*}(0.001)$ \\
ICU & $-0.177(0.116)$ & $-0.060(0.098)$ \\
Service & $-1.193^{*}(0.065)$ & $-0.914 *(0.056)$ \\
Charlson & $-0.046^{*}(0.005)$ & $-0.010^{*}(0.004)$ \\
CTScan & $-0.538^{*}(0.027)$ & $-0.162^{*}(0.023)$ \\
$\alpha$ & $1.828(0.016)$ & \\
$\sigma^{2}$ & $0.960(0.023)$ & $0.291(0.018)$ \\
LogL & -35245.5 & -34423.1 \\
\hline
\end{tabular}

a Significance at the 1,5 and $10 \%$ level is indicated with *,** and $* * *$, respectively. Standard errors are given in parentheses

b For the PCHM seven parameters (not reported) were estimated along with all the other coefficients 
Table 7 Competing risks models: transferred ${ }^{\mathrm{a}}$

\begin{tabular}{lll}
\hline Variables & Weibull with Gamma het. and hospital FE & PCHM with Gamma het. and hospital FE \\
\hline Female & $-0.211^{*}(0.046)$ & $-0.067^{*} *(0.034)$ \\
Age & $-0.061^{*}(0.001)$ & $-0.015^{*}(0.001)$ \\
ICU & $-0.631(0.441)$ & $-0.269(0.324)$ \\
Service & $-1.986^{*}(0.186)$ & $-1.252^{*}(0.137)$ \\
Charlson & $-0.064^{*}(0.009)$ & $-0.013^{* *}(0.007)$ \\
CTScan & $-0.835^{*}(0.060)$ & $-0.260^{*}(0.044)$ \\
$\alpha$ & $1.005(0.022)$ & \\
$\sigma^{2}$ & $2.308(0.169)$ & $0.000(0.000)$ \\
LogL & -13044.7 & -11538.4 \\
\hline
\end{tabular}

a Significance at the 1,5 and $10 \%$ level is indicated with *,** and ***, respectively. Standard errors are given in parentheses

b For the PCHM seven parameters (not reported) were estimated along with all the other coefficients

Table 8 Competing risks models: discharged death ${ }^{\mathrm{a}}$

\begin{tabular}{lll}
\hline Variables & Weibull with Gamma het. and hospital FE & PCHM with Gamma het. and hospital FE \\
\hline Female & $0.078(0.073)$ & $-0.097^{\mathrm{b}}(0.023)$ \\
Age & $-0.024^{*}(0.002)$ & $0.026^{*}(0.001)$ \\
ICU & $0.083(0.347)$ & $0.510^{*}(0.111)$ \\
Service & $-1.551^{*}(0.233)$ & $-0.230^{*}(0.065)$ \\
Charlson & $-0.216^{*}(0.017)$ & $-0.064^{*}(0.005)$ \\
CTScan & $-2.863^{*}(0.120)$ & $-0.725^{*}(0.033)$ \\
$\alpha$ & $2.577(0.105)$ & \\
$\sigma^{2}$ & $17.684(1.054)$ & $0.000(0.000)$ \\
LogL & -25387.1 & -24499.8 \\
\hline
\end{tabular}

a Significance at the 1,5 and $10 \%$ level is indicated with *,** and ***, respectively. Standard errors are given in parentheses

b For the PCHM seven parameters (not reported) were estimated along with all the other coefficients

of its Weibull counterpart and, in the case of two of the risks, transfer and death, it becomes very small suggesting that with a more flexible baseline hazard the covariates are able to capture most of the variability in the data. Indeed, the results for the more flexible model make sense. Contrary to the previous model, gender is now a significant factor in the conditional probability of death. Also, the sign for the effect of age on the conditional probability of death is positive and significant, as expected.

\section{Conclusions}

The literature on length of stay and hospital outcomes is often used to inform policy regarding hospital payment and outcomes. Fenn and Davies (1990) were the first to call attention to the inadequacy of ordinary least square estimation of linear and log-linear models of length of stay and suggest the use of duration (survival) models. Cutler (1995), Hamilton et al. (1996), Ho et al. (2000), and Picone et al. (2003) advanced the literature in 
focusing not only on the importance of accounting for correlation between length of stay and outcomes, but also to control for the potential problem of unobserved patient heterogeneity.

Using a sample of inpatient claims for one DRG, we confirm the observations of Hamilton et al. (1996) that results are sensitive to the use of survival or competing risk models, as well as controls for unobserved patient and hospital heterogeneity.

We did not intend to explore the full range of estimation techniques available for dealing with the modeling of length of stay. Instead, we focused on more standard approaches that can be implemented with relative ease. Our results suggest that researchers should carefully consider the specification of the baseline hazard and rely on more flexible models such as the PCHM, consistent with the findings of Clark and Ryan (3). Unobserved patient and hospital heterogeneity should not be neglected as it can lead to substantial distortions in the results. More importantly, researchers that model length of stay should carefully consider the problem of multiple destinations and estimate competing risk models whenever appropriate.

Acknowledgements The first two authors gratefully acknowledge financial support by the Portuguese Foundation for Science and Technology (FCT; refs. SFRH/BD/5054/2001; POCT/ECO/34666/2000). This revised version has benefited considerably from comments from the editor and two anonymous referees.

\section{References}

Allison, P.D.: Event History Analysis. Regression for Longitudinal Event Data. Beverly Hills, Sage (1984)

Bedford, T., Lindqvist, B. H.: The identifiability problem for repairable systems subject to competing risks. Adv. Appl. Prob. 36, 774-790 2004

Burns, L., Wholey, D.R.: The effects of patient, hospital and physician characteristics on length of stay and mortality. Med. Care 29(3), 251-271 (1991)

Cameron, A.C., Trivedi, P.K.: Microeconometrics: methods and applications. Cambridge, Cambridge University Press (2005)

Canoodt, L.J., Knickman, J.R.: The effect of hospital characteristics and organizational factors on pre- and prospective lengths of hospital stay. Health Serv. Res. 19(5), 561-585 (1984)

Charlson, M.E., Pompei P., Ales K.L.: A new method of classifying prognostic comorbidity in longitudinal studies: development and validation. J. Chronic Dis. 40(5), 373-383 (1987)

Clark, D.E., Ryan, L.M.: Concurrent prediction of hospital mortality and length of stay from risk factors on admission. Health Serv. Res. 37(3), 631-645 (2002)

Clarke, A., Rosen, R.: Length of stay. How short should hospital care be? Euro. J. Public Health 11(2), 166$170(2001)$

Clegg, L., Cai, J., Sen, P., Kupper, L.: Misspecification of marginal hazard models in multivariate failure time data. Sankhya: Indian J. Stat. 62, 25-42 (2000)

Cox, D.R.: The analysis of exponentially distributed lifetimes with two types of failure. J. R. Stat. Soc. Ser. B 21, 411-421 (1959)

Culebras, A., Case, C.S., Masdeu, J.C., Fox, A.J., Bryan, R.N., Grossman, C.B., Lee, D.H., Adams, H.P., Thies, W.: Practice Guidelines for the Use of Imaging in Transient Ischemic Attacks and Acute Stroke A Report of the Stroke Council American Heart Association. Stroke 28(7), 1480-1497 (1997)

Cutler, D.M.: The incidence of adverse medical outcomes under prospective payment. Econometrica 63(1), 29-50 (1995)

David, H.A., Moeschberger, M.L.: The Theory of Competing Risks. London, Griffon (1978)

Dismuke, C.E., Guimaraes, P.: Has the caveat of case-mix based payment influenced the quality of inpatient hospital care in Portugal? Appl. Econ. 34, 1301-13070 (2002)

Elbers, C., Ridder, G.: True and spurious duration dependence: the identifiability of the proportional hazard model. Rev. Econ. Stat. 49(3), 403-409 (1982)

Fenn, P., Davies, P.: Note: variations in length of stay. J. Health Econ. 9(2), 223-243 (1990)

Gooley, T.A., Leisenring W., Crowley J., Storer B.E.: Estimation of failure probabilities in the presence of competing risks: new representations of old estimators. Stat. Med. 18(6), 695-706 (1999) 
Hamilton, B.H., Hamilton, V.H., Mayo, N.E.: What are the costs of queuing for hip fracture surgery in Canada? J. Health Econ. 15(2), 161-185 (1996)

Hamilton, B.H., Hamilton, V.: Estimating surgical volume-outcome relationships applying survival models: accounting for frailty and hospital fixed effects. Health Econ. 6(4), 383-395 (1997)

Heckman, J., Singer, B.: A method for minimizing the impact of distributional assumptions in econometric models for duration data. Econometrica 52(2), 271-320 (1984)

Heckman, J.J., Honoré, B.E.: The identifiability of the competing risks model. Biometrika 76(2), 325-330 (1989)

Ho, V., Hamilton, B., Roos, L.L.: Multiple approaches to assessing the effects of delays for hip fracture patients in the United States and Canada. Health Serv. Res. 34(7), 1499-1518 (2000)

Hougaard, P.: Life table methods for heterogeneous populations: distributions describing the heterogeneity. Biometrika 71(1), 75-83 (1984)

Jones. A.: Health econometrics. In: Newhouse, J.P., Culyer, A.J. (eds.) Handbook of Health Economics. Amsterdam: The Netherlands (2000)

Lancaster, T.: The Econometric Analysis of Transition Data. Cambridge, Cambridge University Press (1990)

Patient Classification Systems Europe. Country Update. 19th Patient Classification Systems Europe Conference, Washington DC, 08.-11. October 2003.

Picone, G., Wilson, R.M., Chou, S.Y.: Analysis of hospital length of stay and discharge destination using hazard functions with unmeasured heterogeneity. Health Econ. 12(12), 1021-1034 (2003)

Prentice, R.L., Kalbfleisch, J.D., Peterson, A.V., Flournoy, N., Farewell, V.T., Breslow, N. E.: The analysis of failure times in the presence of competing risks. Biometrics 34, 541-554 (1978)

Rodriguez, G.: 2000. Survival Analysis. Available at: http://data.princeton.edu//wws509/notes/c7.pdf

Shi, L.: Patient and hospital characteristics associated with average length of stay. Health Care Manage. Rev. 21(2), 46-61 (1996)

Therneau, T.M., Grambsch, P.M.: Modeling Survival Data: Extending the Cox Model. New York, Springer (2000) 